\title{
Dyshidrosiform pemphigoid localized on the hands in a child: a rare occurrence*
}

\author{
Surabhi Dayal ${ }^{1}$ \\ Vijay Kumar Jain ${ }^{1}$
}

\author{
Priyadarshini Sahu
}

DOI: http://dx.doi.org/10.1590/abd1806-4841.20174949

\begin{abstract}
Dyshidrosiform pemphigoid is an acquired autoimmune variant of bullous pemphigoid with persistent vesicobullous eruptions localized on the palms or soles, or both. It generally occurs in the elderly and is rarely reported in childhood. Hereby, we describe the first case of dyshidrosiform pemphigoid in a 12-year-old child, which was limited to the dorsal hands and treated successfully with dapsone (diaminodiphenyl sulfone). Along with this report, we also review the clinical features of various types of dyshidrosiform pemphigoid.
\end{abstract}

Keywords: Child; Dapsone; Pemphigoid, bullous

\section{INTRODUCTION}

Dyshidrosiform pemphigoid (DP) is an infrequent variant of localized pemphigoid that was first described by Levine $e t$ al. in a 72-year-old male in 1979. ${ }^{1}$ Since then only 30 cases of DP have been reported in the literature, mainly affecting the elderly. Herein, we report a rare case of dyshidrosiform bullous pemphigoid in a 12-yearold child that was resistant to steroids with recurrent vesicobullous eruptions on the dorsal aspects of the hands. He responded very well to dapsone within 3 months, without recurrence 6 months after discontinuing treatment treatment.

\section{CASE REPORT}

A 12-year-old male child presented to our outpatient department of dermatology with recurrent multiple itchy fluid-filled lesions located on both hands for 18 months. These lesions were painful and ruptured in 4-5 days, leaving raw areas, which gradually healed without scarring. Multiple such episodes occurred in the last 18 months. His developmental, past, and family histories were noncontributory. He denied consistent exposure to allergens or any oral medication before the onset of the bullous lesions.

On examination, the patient showed multiple tense vesicles and bullae of various sizes, with a base of normal skin on the dorsum of both hands (Figure 1). No mucosal or other cutaneous involvement was observed. Nikolsky's sign was negative. Potassium hydroxide preparation and bacterial and fungal cultures were negative.
Blood biochemistry tests were within normal limits. A clinical diagnosis of pompholyx had been made earlier by a private practitioner, who prescribed oral prednisolone, antihistamines, and potent topical steroids in the last year. This treatment was associated with mild improvement and recurrent exacerbation of lesions after a tapering dose of steroids.

We performed a skin biopsy from a representative lesion on the left hand with the differential diagnosis of DP, pompholyx, irritant contact dermatitis, epidermolysis bullosa acquisita (EBA), and bullous impetigo. Biopsy specimens revealed a subepidermal vesicle with a dermal infiltrate consisting predominantly of eosinophils (Figure 2). Direct immunofluorescence was positive for continuous linear deposition of immunoglobulin $\mathrm{G}$ (IgG) at the dermoepidermal junction (Figure 3). We were unable to perform indirect immunofluorescence (IIF).

Based on clinical features and biopsy, we reached a diagnosis of DP. Since IIF could not be performed, a diagnosis of EBA could not be excluded. However, the early age of onset in our patient (12 years), the type of lesions that were conspicuously not associated with scarring, milia formation and hyperpigmentation upon healing, and the prompt response to dapsone favored the diagnosis of DP over EBA. The patient was started on dapsone 50mg daily, which produced a rapid improvement with the reduction of new lesion formation in a month's time. After 2 months of treatment, dapsone was tapered ( $25 \mathrm{mg}$ daily) and stopped with a complete clinical

Received on 18.07.2015.

Approved by the Advisory Board and accepted for publication on 10.10.2015.

* Work performed at the Department of Dermatology, venereology and leprology, Pt B D Sharma Post Graduate Institute of Medical Sciences, Rohtak - Haryana, India

Financial support: none.

Conflict of interest: none.

Pt. B. D. Sharma, Post Graduate Institute of Medical Sciences - Rohtak- Haryana, India.

Shaheed Hasan Khan Mewati, GMC, Nalhar, Haryana, India

(C)2017 by Anais Brasileiros de Dermatologia 
remission after 3 months from the onset of lesion formation. Now, 6 months later, the patient is still in complete remission with no new lesions after stopping dapsone.

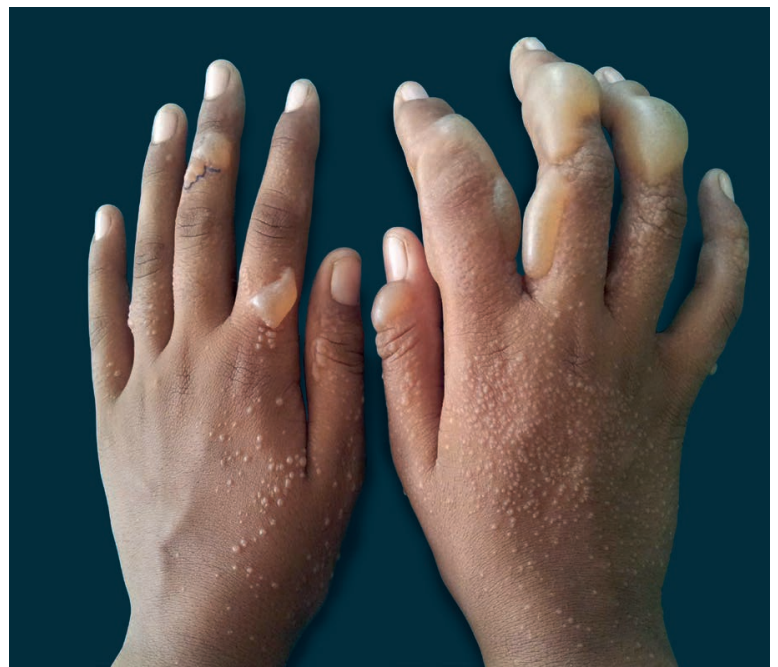

FigURE 1: Multiple tense vesiculobullous lesions on the bilateral dorsal hands

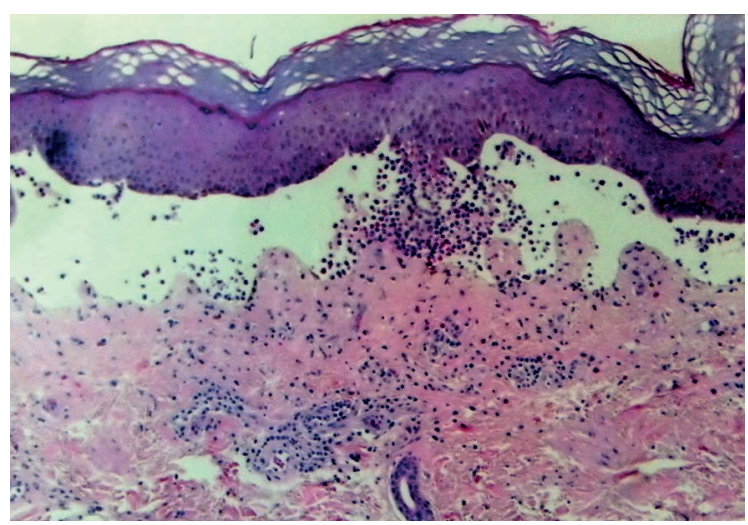

Figure 2: Histological examination of a vesicle reveled a subepidermal vesicle with infiltration of eosinophils (Hematoxylin \& eosin X200)

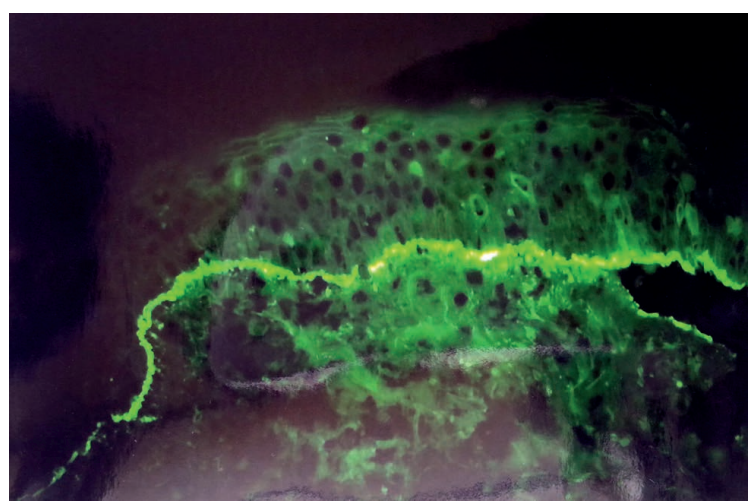

Figure 3: Direct Immunofluorescence test shows linear deposits of IgG at the dermo-epidermal junction (X200)

\section{DISCUSSION}

Bullous pemphigoid (BP) is an acquired autoimmune blistering disease that is characterized by subepidermal blisters. Dyshidrosiform pemphigoid is a localized variant of BP, clinically characterized by vesicobullous lesions localized to either palmar or plantar surfaces, or both. Although this is usually the only feature, it may occasionally progress to generalized BP. It is usually associated with autoantibodies directed against hemidesmosomes in the basement membrane zone: the $180-\mathrm{kDa}$ BP antigen (a transmembranous molecule) and the 230-kDa BP antigen (an intracellular molecule). ${ }^{2}$ Because DP shares the same causative antibody with BP, histological and immunofluorescence findings of DP are consistent with those of BP. However, the DP mechanism is unclear.

DP predominately affects the elderly, but sometimes appears in children under 18 years of age. The literature includes 30 cases of DP (including the childhood cases), involving the palmoplantar area and may be treated according to different clinical courses. According to Caldarola et al. ${ }^{3}$, these cases can be divided into 3 groups:

1) Group I: vesiculobullous lesions localized in the plamoplantar region (described in only 9 cases).

2) Group II: palmoplantar eruption prior to classical BP eruption on the rest of the body by weeks or months (7 cases).

3) Group III: palmoplantar lesions appeared along with generalized bullous eruption. (14 cases).

Caldarola et al. proposed that a diagnosis of DP may be applied only to Group I \& II patients. Group III is defined as BP with simultaneous palmoplantar involvement, which is not rare, particularly in the pediatric age group. Of the 30 cases investigated in the literature, we found only 6 of childhood BP associated with the involvement of palms and soles along with generalized body involvement. We found no cases of childhood BP with localized palm and sole involvement. As the palm and sole lesions are also associated with concomitant involvement of other cutaneous sites, these cases were classified under Group III. Our case was unique because the lesions were limited to the dorsum of the hands, thus categorically falling under Group I. After a thorough review of the literature, our case seems to be the first case of DP in a 12-year-old child that belongs to Group I, i.e., involvement localized only to hands.

This localized variant of DP in adults is very responsive to steroids and is usually associated with marked improvement and remission. The prognosis of childhood BP is generally better as compared to the adult type. ${ }^{4}$ However, in some cases of childhood BP, the skin lesions relapse and require prolonged treatment or adjuvant therapies such as dapsone, azathioprine, cyclosporine, or erythromycin/niacinamide. ${ }^{5}$ Similarly, in our case, the lesions on the dorsum of the hand were resistant to treatment with oral and topical steroids and responded dramatically to dapsone within 3 months. This successful treatment with dapsone prevented the child from lesion spread and from the long-term side effects of corticosteroids such as growth retardation. Thus, to our knowledge, this is the first case of dyshidrosiform childhood bullous pemphigoid that obtained remarkable improvement by the administration of dapsone alone, without recurrence after 6 months of follow up. $\square$ 


\section{REFERENCES}

1. Levine N, Freilich A, Barland P. Localized pemphigoid simulating dyshidrosiform dermatitis. Arch Dermatol. 1979;115:320-1.

2. Diaz LA, Ratrie H 3rd, Saunders WS, Futamura S, Squiquera HL, Anhalt GJ, et al. Isolation of a human epidermal cDNA corresponding to the 180-kDautoantigen recognized by bullous pemphigoid and herpes gestationis sera. Immunolocalization of this protein to the hemidesmosome. J Clin Invest. 1990;86:1088-94.

3. Caldarola G, Fania L, Cozzani E, Feliciani C, De Simone C. Dyshidrosiform pemphigoid: a well-defined clinical entity? Eur J Dermatol. 2011;21:112-3.

4. Waisbourd-Zinman O, Ben-Amitai D, Cohen AD, Feinmesser M, Mimouni D, Adir-Shani $A$, et al. Bullous pemphigoid in infancy: clinical and epidemiologic characteristics. J Am Acad Dermatol. 2008;58:41-8.

5. Motegi S, Abe M, Tamura A, Ishii N, Hashimoto T, Ishikawa 0. Childhood bullous pemphigoid successfully treated with diaminodiphenyl sulfone. J Dermatol. 2005;32:809-12.
MAILING ADDRESS:

Priyadarshini Sahu

432, Sector-IV, R. K. Puram

New Delhi- 110022

E-mail: priyadarshini.sahu.9@gmail.com

How to cite this article: Dayal S, Sahu P, Jain VK. Dyshidrosiform pemphigoid localized on the hands in a child: a rare occurence. An Bras Dermtatol. 2017;92(5): 714-6. 